\begin{tabular}{ccc}
\hline & International Journal of Engineering \& Technology, $7(2.6)(2018) 24-27$ \\
SPC & International Journal of Engineering \& Technology \\
\hline
\end{tabular}

\title{
Air quality and water quality monitoring using XBee and internet of things
}

\author{
Anita Gehlot ${ }^{1,3}$, Rajesh Singh ${ }^{1,3}$, Rohit Samkaria ${ }^{1,3}$, Sushabhan Choudhary ${ }^{1,3}$, Aisik De ${ }^{2}$, Kamlesh ${ }^{2}$ \\ ${ }^{1}$ Department of Electronics Instrumentation and Control Engineering \\ ${ }^{2}$ Department of mechanical Engineering \\ ${ }^{3}$ UPES University Dehradun India \\ *Corresponding author E-mail: anita@ddn.upes.ac.in
}

\begin{abstract}
There are numbers of disadvantage in the current existing systems for water quality monitoring which are generally limited to only a few varieties of parameter provide when there and need to be sending through manual operation. This type of security systems required a device which could send alert signal to the sub - stations and is very time consuming process. The quick warning or alert is not possible in the current existing system because they need some manual operation to be performed which could send the data and this cause a huge casualty because sometimes if the area is not in taken care of then it may cause huge causality to human as well as marine habitats. For such conditions a system required which automatically reads the sensor values (parameters) compares them with the threshold of fresh air and water parameters and if this level is above the threshold then automatically send alert with the pin point location to the sub - stations.
\end{abstract}

Keywords: WSN; Internet of Things (IoT); Xbee; Thingspeak.

\section{Introduction}

About $70 \%$ of the Earth's surface is covered with water and the rest of it is land, out of which $99 \%$ of water is the ocean water which is not it for drinking, and the $1 \%$ which is left consists of lakes, rivers, ground water is continuously being polluted by humans. Zang Y et al. has evaluated using national air and water quality data layers at both water management catchment and Robust farm Type [1]. This is the form of environmental degradation occurs when pollutants directly or indirectly discharged into water bodies without removal of harmful compounds such as detergents by-products, chloroform, food processing waste, pesticides and etc. These waste substance causes contamination of water and make it unfit for drinking which gives rise to various diseases such as various forms of water borne diarrheal diseases are the most prominent example. Water borne diseases are caused by pathogenic microorganisms that are commonly transmitted through contaminated fresh water. Physical contact with the contaminated water can cause skin infections. Pollution disrupts the natural food chain most of the water predators dies due to the contamination of their habitat - dead fishes, crabs, birds and sea gulls, dolphins and many other often wind up on the beaches, killed by the pollutants in their habitat. Ozone Measurement is critical component of air quality so Pang Xiobing et. al. study the electrochemical $\mathrm{O} 3$ sensor in a miniaturized $\mathrm{O} 3$ measurment device combined with Labjack and LabView data acquisition [2]. The objective methodology for determining the optimum number of ambient air quality monitoring that suggest the two fold approach is a combination of fuzzy similarity measures and fuzzy cman clustering [3]. An array of three long period gratings that is fabricated in single optical fiber and multiplexed in the wavelength domain was used measure humidity, temperature, volatile organic compound that are key indoor air quality indicators [4]. Wireless Sensor Networks are emerging the latest technologies for sensing, measuring the pollutant levels and transmit the information in the critical server [6]. With the advent of internet of things that emerged simultaneously with development of data acquisition has been utilizing the best part in water and air quality monitoring systems [7], [8].

\subsection{Causes of water pollution}

- Dumping solid wastes and littering by humans in rivers, lakes and oceans. Littering items include cardboard, Styrofoam, aluminium, plastic and glass.

- Oil Pollution caused by oil spills from tankers and oil from ship travel. Oil does not dissolve in water and forms a thick sludge.

- Sewage from domestic households, sewage can be more problematic when people flush chemicals and pharmaceutical substances down the toilet.

Air pollution equally contributes to the contamination of water bodies and effect the food chain adversely. Nitrogen compounds in air pollution are partly the cause of algal blooms, and can also contributes to acidic nature of the water bodies thus causing in misbalance in health and environment as well. In simple words air pollution is the introduction of harmful substances including particulates and biological molecules into Earth's atmosphere. It may cause diseases, allergies or cause death of any species. Air pollution can aggravate the medical condition of individuals with asthma and emphysema, long term health effects can include chronic respiratory disease, lung cancer, heart disease, and even damage to the brain, nerves, liver or kidneys.

\subsection{Causes of air pollution}

- Emissions from industries and manufacturing activities. 
- Burning of fossil fuels. Motor vehicles produce high levels of Carbon oxides (COx) and Hydrocarbons (HC) and Nitrogen Oxides ( $\mathrm{NxOy})$.

- Household and farming chemicals. (crop dusting, fertilizers dust emits harmful chemicals into the air)

- Deforestation is also a main cause that contributes to air pollution and green - house effect.

\section{Proposed system}

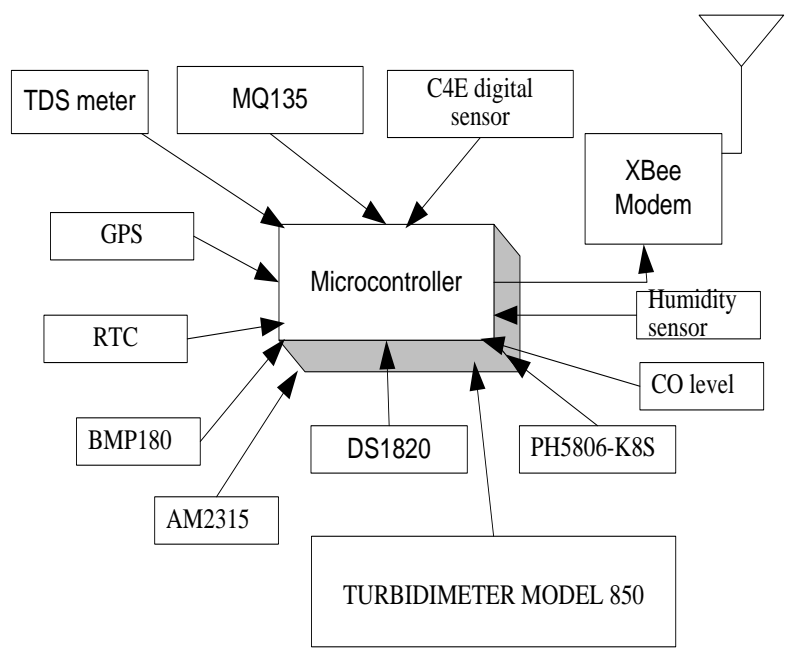

Fig. 1: Schematic Architecture of Proposed System.

The proposed work is achieved by

- Designing a system that that reads the various parameters of the river water and simultaneously transmitting the parameters to the respective nodes.

- The system also monitors the air quality by monitoring the various contaminants including carbon oxides (COx), nitrogen oxides (NOx), sulphur oxide (SOx) and hydrogen sulphides (HxSy).

- The system can be used for ad hoc as well as continuous monitoring of the particular area.

- Low-cost sensing using printing of transducers on cheap, flexible, benign substrates.

- Develop alert system in case of high pollution episodes.

- Develop air quality forecast systems and procedures.

- As a result, the receiving station will be informed about the water as well as the air quality of that particular location. The pin - point location of the area will be transmitted using GPS to the receiving station.

- Design and set up a standardized data transfer and data processing system that enables a free flow of information between cities, central government and eventually the public.

- To develop a database for future pollution control strategies. The schematic architecture consists of centralized microcontroller unit that read the data of various sensors and transmit this data to the base station through the Xbee wireless protocol. The proposed approach is to sense the various contaminants around the river water system and transmits the data to the stations in real - time and the sensor performance and the loss calibration will be rectified with time.

\section{System description}

The proposed design divided into three main parts: -

\subsection{Mobile system}

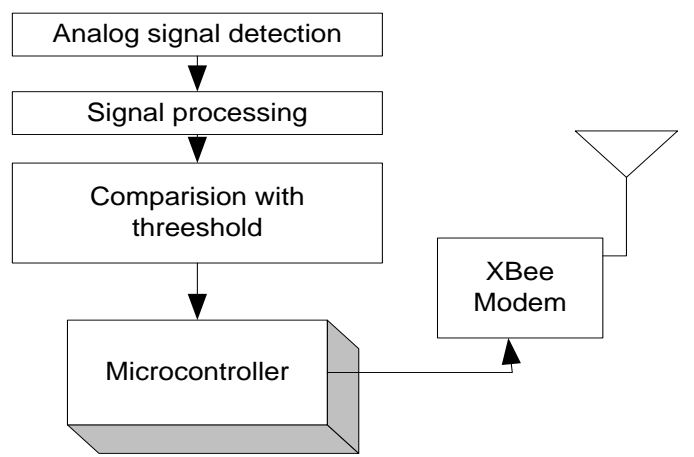

Fig. 2: Block Diagram of Mobile System.

It is a system that consist of all the required sensors on it which compares the value of the calculated parameters to the prerequisite value of the threshold parameters and if the level exceeds it will intimate the subsystem about all the collected data and compares the data simultaneously.

\subsection{Sensor subsystem}

Turbidity is the main parameter that is to be measured, we will be using TURBIDIMETER MODEL 850 to measure turbidity as it uses an insertion type sensor. PH5806-K8S pH electrode will be used to measure the $\mathrm{pH}$ of the water system to know the characteristics of the water system. AM2315 Encased I2C Temperature/Humidity sensor is used to measure the temperature and humidity of the water stream and compare it with the threshold. C4E digital sensor will be used to monitor the salinity of the surface water. DS18B20 will be interfaced to record the water temperature in real time.

On the other hand for air quality monitoring we have SDS011 to monitor air particle (dust particle) PM2.5/PM10, and MQ135 which is used in our air quality monitoring equipment which are suitable of detecting NH3, NOx, various alcohols, smoke, benzene and COx also and an RTC DS1307 on the monitoring system.

\subsection{Sub-station}

Sub - station will be the primary unit of this project which will receive the data in the first hand and analyse, also compares the received data independently and gives the updates to the main station by uploading the information on cloud which will be easily monitored by the control room.

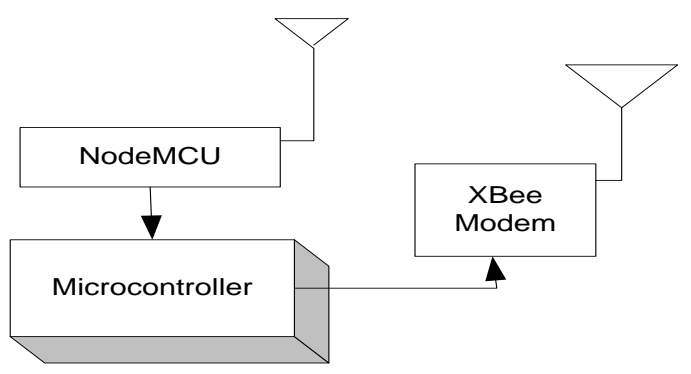

Fig. 3: Architecture of Sub-Station.

\subsection{Central control room}

The central control room will be notified about the situation and will take action accordingly as per the situation of the area recorded, and with the help of this team work a safe environment could be achieved. 


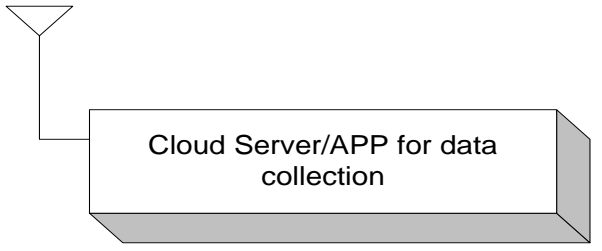

Fig. 4: Central Monitoring.

\section{Design and development of system}

For the project, design of the drone that will go in the water system and monitors various parameters. Designing of the drone that do locomotion on water as well as land is a typical task in this project, for the development of the same, expertise from the industries as well as academia will be sought. The system embedded on the mobile unit will record the various parameters as discussed above and transmits them though antenna.

The system designing includes the development of algorithms for the various sensors and then their interfacing, the Sensors - Subsystem which fed this data to the controller unit through which decision taken process is controlled. There the number of platform incorporates within the whole system which ultimate limelight the product in a well-designed and effective shape. The designing of the product will be done through step by step:

- Initial task is to develop the Sensor Subsystem unit to monitor the threshold level of various parameters necessary for air and water quality monitoring.

- Decision making capability is provided by controller unit which booted up by Embedded coding in a hierarchal steps.

- The two conditions provides the next step i.e. if data collected is above threshold level then initialize the GPS and Communication unit track the location and send the location details to the Control Room.

The main impact of the system be considered effective by the power back up of the product. For the long term power backup of the product need to be designed a special battery unit and a specially designed embedded part that consumes a very less power so that the battery could stable for long last.

\subsection{Live data plotting over cloud (thingspeak)}

The real time data is plotted over the cloud using the ThingsPeak that is open source channel provide the facility of Live Plotting of the data over the website. The data is uploaded continuously after duration of $20 \mathrm{~ms}$. The framework for the uploading of the data over the cloud is uploaded to the controller NodeMCU that is developed on Arduino open source platform.

\subsubsection{Air monitoring}

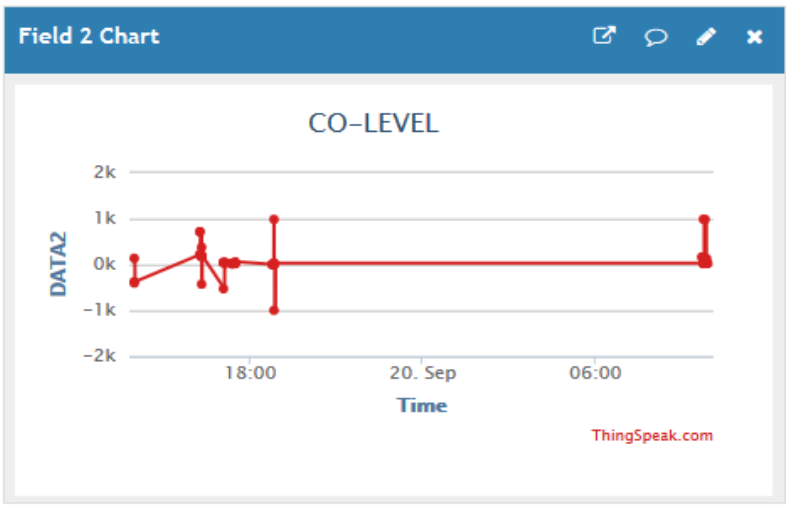

Field 3 Chart

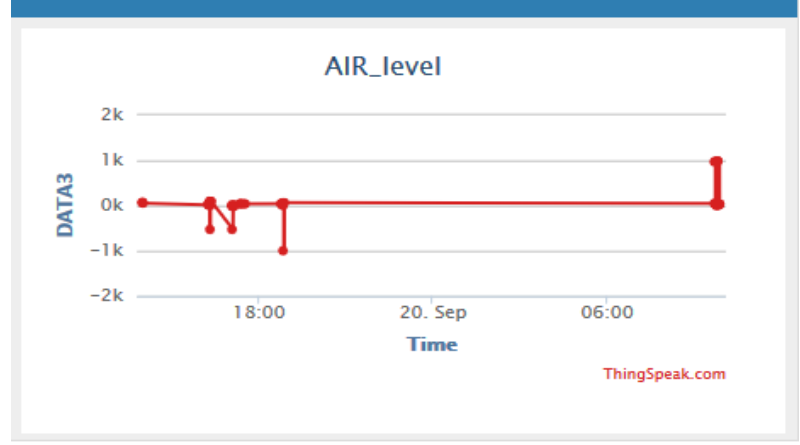

Fig. 5: Live Plotting of Data Over Thing Peak.

Whatever the data collected by the sensor node uploaded to the sever through the substation that is operated with the NodeMCU.

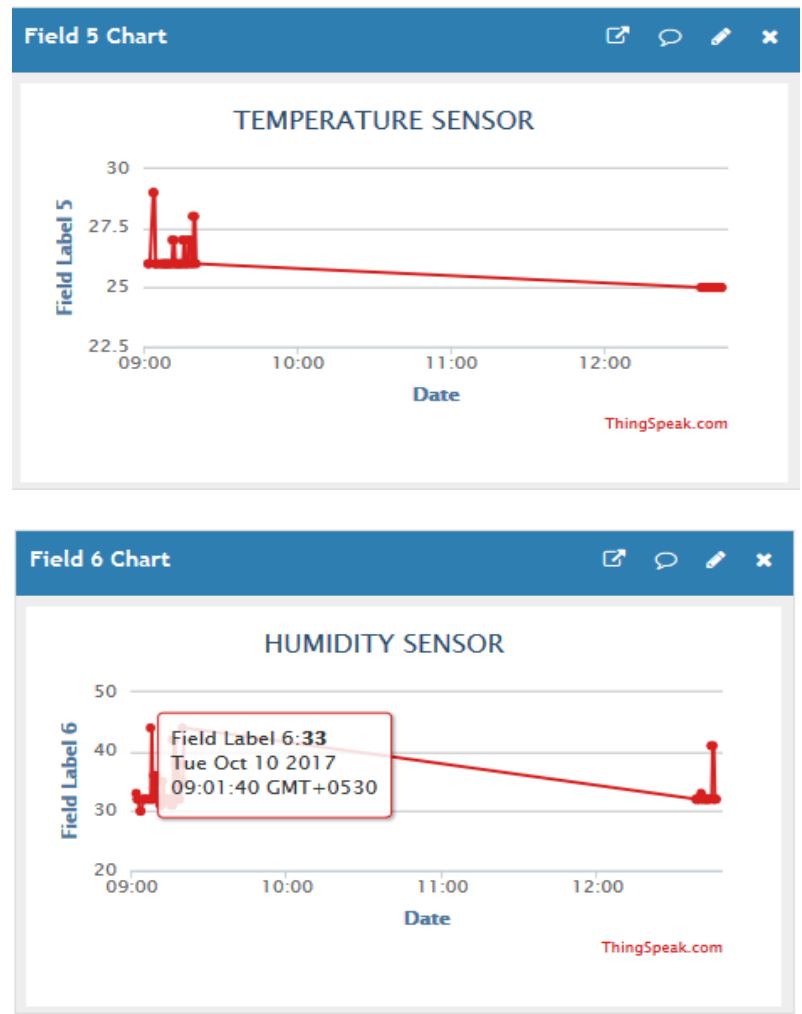

Fig. 6: Live Plotting Temperature and Humidity.

In order to upload data to the server the user need to register in the ThingsPeak site. After registration each user is provided with a unique API key which is the source to upload the data to the site. User can create multiple no. of channels to upload the data and each channel has 8 different fields that can be used to upload the individual sensor data. 

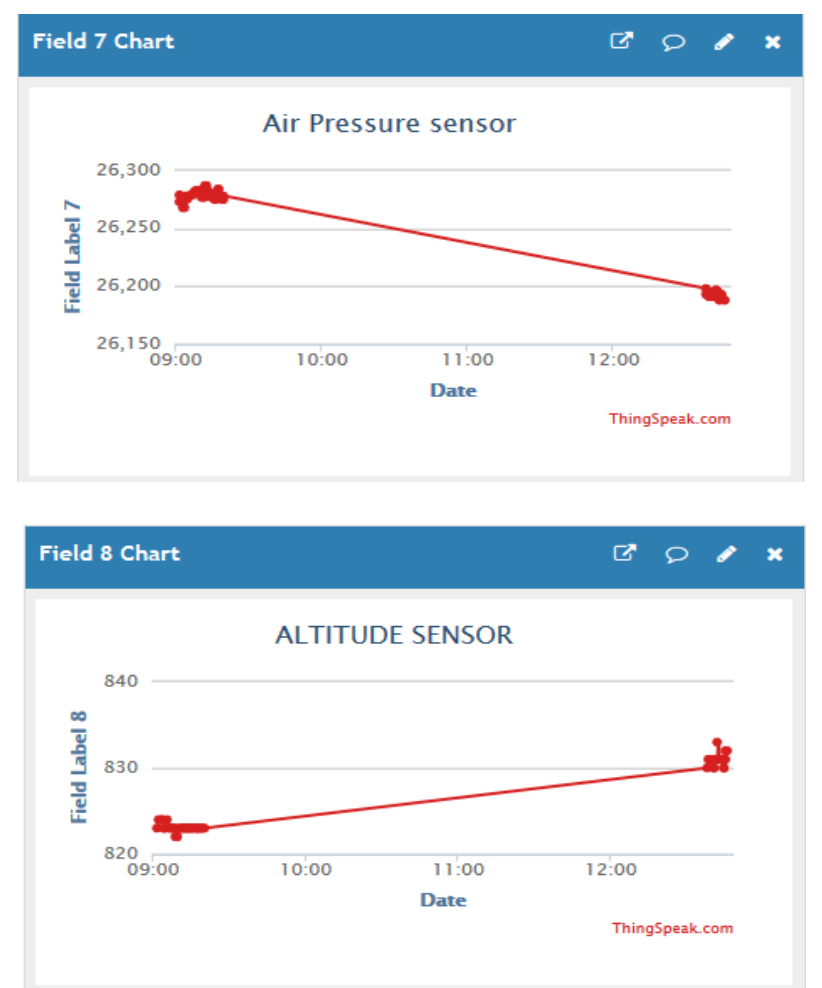

Fig. 7: Live Plotting Air Pressure and Altitude.

\subsubsection{Data of water monitoring system}

Sensor used for the water monitoring basically monitor the TDS level of the water.

\begin{tabular}{ll}
\hline Water & TDS Value \\
\hline From RO, Distillation Pure Drinking water & $0-50$ \\
Mountain Springs, Carbon filtration, Aquifers & $50-170$ \\
Hard Water & $170-300$ \\
High TDS water from Tap or Minerals & $300-500$ \\
EPA,s Maximum Contamination Level & 500 or above \\
\hline
\end{tabular}

\section{Result and conclusion}

This device will be very much helpful for monitoring uses, as there is no need of any one to go in person to check the particular area. Because when a water system starts being contaminated then an alert will automatically be sent to the control room that area threshold in so and so area exceeds the level. By simply establishing the system near the water system, that has to be monitored in the first hand all the needed parameters can be monitored. Through this device can alert the people at any part of the globe if you have an active internet connectivity that would allow you to access the server for the information. This device is helpful in future when any problem arises in water system. The developed system have the features are

- Simple cost effective system for monitoring of a river system.

- It is not limited to the distance barrier; the alert can be through data anywhere in the globe.

- Simple and customized usage of embedded systems which may help to send alert or warning automatically.

- Proper usage of technology for River Water Monitoring.

- Monitoring of various parameters to their threshold.

- The sensor based information that prevents against rodents.

- Automatically alert sending will provide a huge reduction to casualty.

The system will be a huge benefit for humanity even in adverse circumstances where they cannot contact the authority for survey of any water system.

\section{References}

[1] Zhang, Y., et al. "Projected impacts of increased uptake of source control mitigation measures on agricultural diffuse pollution emissions to water and air." Land Use Policy 62 (2017): 185-201. https://doi.org/10.1016/j.landusepol.2016.12.017.

[2] Pang, Xiaobing, et al. "Electrochemical ozone sensors: A miniaturised alternative for ozone measurements in laboratory experiments and air-quality monitoring." Sensors and Actuators B: Chemical 240 (2017): 829-837. https://doi.org/10.1016/j.snb.2016.09.020.

[3] Maji, Kamal Jyoti, Anil Kumar Dikshit, and Ashok Deshpande. "Can fuzzy set theory bring complex issues in sizing air quality monitoring network into focus?." International Journal of System Assurance Engineering and Management 8.4 (2017): 2118-2128. https://doi.org/10.1007/s13198-014-0327-1.

[4] Hromadka, Jiri, et al. "Multi-parameter measurements using optical fibre long period gratings for indoor air quality monitoring." Sensors and Actuators B: Chemical 244 (2017): 217-225. https://doi.org/10.1016/j.snb.2016.12.050.

[5] Islam, Tarikul, Subhas Chandra Mukhopadhyay, and Nagender Kumar Suryadevara. "Smart Sensors and Internet of Things: A Postgraduate Paper." IEEE Sensors Journal 17.3 (2017): 577-584 https://doi.org/10.1109/JSEN.2016.2630124.

[6] Mani, A., A. Manolasya, and C. Nandhini. "Survey on air pollution monitoring system using sensors." International Journal of

[7] Habibi, R., and A. A. Alesheikh. "Quality of Spatial Interpolation Services Assessment for Development of Air Pollution Monitoring Systems Based on the Internet of Things." Journal of Geomatics Science and Technology 6.4 (2017): 1-15.

[8] Firdhous, M. F. M., B. H. Sudantha, and P. M. Karunaratne. "IoT enabled proactive indoor air quality monitoring system for sustainable health management." Computing and Communications Technologies (ICCCT), 2017 2nd International Conference on. IEEE, 2017. https://doi.org/10.1109/ICCCT2.2017.7972281.

[9] Singh, Purnendu Shekhar Pandey Rajesh, and Vinit Kumar Sharma. "ECG Monitoring System in Wireless Personal Area Network simulation and design using Zigbee Transceiver Module for Health Care Solution."

[10] Mano, Hiroyuki, et al. "Water quality comparison of secondary effluent and reclaimed water to ambient river water of southern Okinawa Island via biological evaluation." Environmental Monitoring and Assessment 189.9 (2017): 442. 